\title{
Regenerations- und Heilungsversuche an der Proactinula von Ectopleura dumortieri (Athecatae-Anthomedusae) unter Anwendung der Zeittransformation ${ }^{1}$
}

\author{
Willi KuHL und GerTrud KuHL \\ Institut für kinematische Zellforschung der Universität, Frankfurt a. M., \\ und \\ Biologische Anstalt Helgoland, Meeresstation, Helgoland
}

\begin{abstract}
Regeneration and healing experiments on the proactinula of Ectopleura dumortieri (Athecatae-Anthomedusae) by employing time transformation. Micro-surgical operations were performed on different developmental stages of Ectopleura dumortieri VAN BENEDEN (Tubularidae, Athecatae-Anthomedusae). The tested stages, i.e., 8th cell-division until proactinula ("Sternchenstadium"), revealed a considerable capacity for healing, regeneration and formation of new body parts. After cutting the embryo up - into a maximum of 10 parts - all fragments assumed the shape of a sphere, formed tentacles and, in part, aboral stem-anlagen as well as the bases of the oral tentacles. The fragments usually form twice as many tentacles as do the normal actinula-stages. If the fragments are not too small, polyps are formed which may settle down and take up food. These polyps differ from normal ones only in that they have fewer tentacles. Vitality and locomotory behaviour of regenerates and newly formed body parts were assessed by means of time lapse movie pictures.
\end{abstract}

\section{EINLEITUNG}

Ausreichendes Vorkommen der Medusen von Ectopleura dumortieri vaN BENEDEN (Tubularidae) im Plankton während des Monats August 1966 ermöglichte die Aufzucht der Eier über das "Sternchen-Stadium" (Proactinula) bis zum Polypen. Es ergab sich damit die Gelegenheit, die Potenzen der eigenartigen Proactinula in bezug auf das Wundheilungs- und Regenerationsvermögen am lebenden Objekt zu prüfen und das Bewegungsverhalten unter $\mathrm{Z}$ e it r a f $\mathrm{f}$ u $\mathrm{g}$ (Z.R.) zu untersuchen. Die normale Entwicklung des Ectopleura-Eies ist von KuHL \& KuHL (1967) beschrieben worden.

\section{UNTERSUCHUNGSMETHODEN}

Die zu den Versuchen verwandten Entwicklungsstadien wurden in filtriertem Seewasser aus befruchteten Eiern gezüchtet. Die mikrochirurgischen Eingriffe erfolgten widmet.

${ }^{1}$ Hierrn Professor Dr. FrIedrICH KRÜGRR zum 65. Geburtstag am 18. August 1967 ge- 
unter dem Binokularmikroskop mit Hilfe feiner Rasierklingen-Fragmente, die in bestimmtem Neigungswinkel an Glasstäbchen geklebt wurden. Das Verhalten der operierten Entwicklungsstadien von Ectopleura wurde meist sofort nach dem Eingriff in geeigneten Mikroaquarien unter Z.R. aufgenommen und damit dokumentarisch festgehalten. Die Zeittransformation schwankte je nach dem Bewegungsverhalten und der Geschwindigkeit des Ablaufs der Heilungs- und Regenerationsvorgänge zwischen einer Z.R. auf $1 / 8$ bis 1/640. Die Aufnahmen erfolgten mit der Kombination Arriflex-16Kamera-Ortholux-II-Mikroskop-Aristophot-III von der Firma E. Leitz (KurL 1966). Als Ergänzung dienten Mikroaufnahmen auf Kleinbildformat $24 \times 36 \mathrm{~mm}$. Eine Teilbild-Analyse der Regenerationsvorgänge ist bei diesen Versuchen infolge der bald nach der Herstellung der Keimfragmente einsetzenden Kontraktionen der auswachsenden Tentakel und der dadurch bedingten Ortsverlagerungen nicht möglich.

\section{BEFUNDE}

\section{Heilung von Schnittwunden}

Zur Vermeidung von Wiederholungen werden im folgenden Einzelheiten der Versuche sowie Zeitangaben in die ausführlicher gehaltenen Legenden zu den Abbildungen verlegt.

Das mit 11 Tentakeln versehene "Sternchenstadium" der Abbildung 1 ist in der Lage, einen über die Mitte des scheibenförmigen Körpers zwischen zwei Tentakeln geführten Schnitt in 2 Stunden und 8 Minuten (c) auszuheilen. Trotz der erheblichen Verletzung wird das Auswachsen der Tentakel ohne Störung weitergeführt. Die Anlage des Polypenstiels in der Mitte der Proactinula, in (b) bereits angedeutet, ist wieder völlig intakt, obwohl der Schnitt hindurchging; (d) gibt einen Eindruck der lebhaften Tentakelbewegungen (unter Z.R.) der normal ausgebildeten Proactinula (5 Stunden 23 Minuten nach der Operation).

Bei dem sehr weit geführten Einschnitt in die Proactinula der Abbildung 2 (b) sind 3 der 12 Tentakel zerstört. 1 Stunde 6 Minuten nach der Verletzung ist neben der Schnittstelle ein neuer Tentakel entstanden, der relativ schnell auswächst (d). 7 Stunden 47 Minuten nach der Verletzung ist auch bei diesem Versuch eine n o r male Proactinula entwickelt mit 10 langgestreckten beweglichen Tentakeln. An Stelle der drei beim Eingriff zerstörten Tentakel ist also nur einer neu ausgebildet worden, beziehungsweise aus dem in Abbildung 2 (b) rechts neben der Schnittstelle erkennbaren „Stumpf“ (= ursprüngliche Tentakelanlage?) hervorgegangen. Die Stielanlage auf der aboralen Seite (f) ist deutlich ausgeprägt; sie ist an der Schnittstelle leicht „eingedellt".

\section{Entfernung sämtlicher Tentakel der Proactinula}

Der in Abbildung 3 in 8 Teilbildern einer Z.R.-Aufnahme dargestellte Versuch zeigt die Fähigkeit der Proactinula, „neue" Tentakel auszubilden, wenn die bereits 


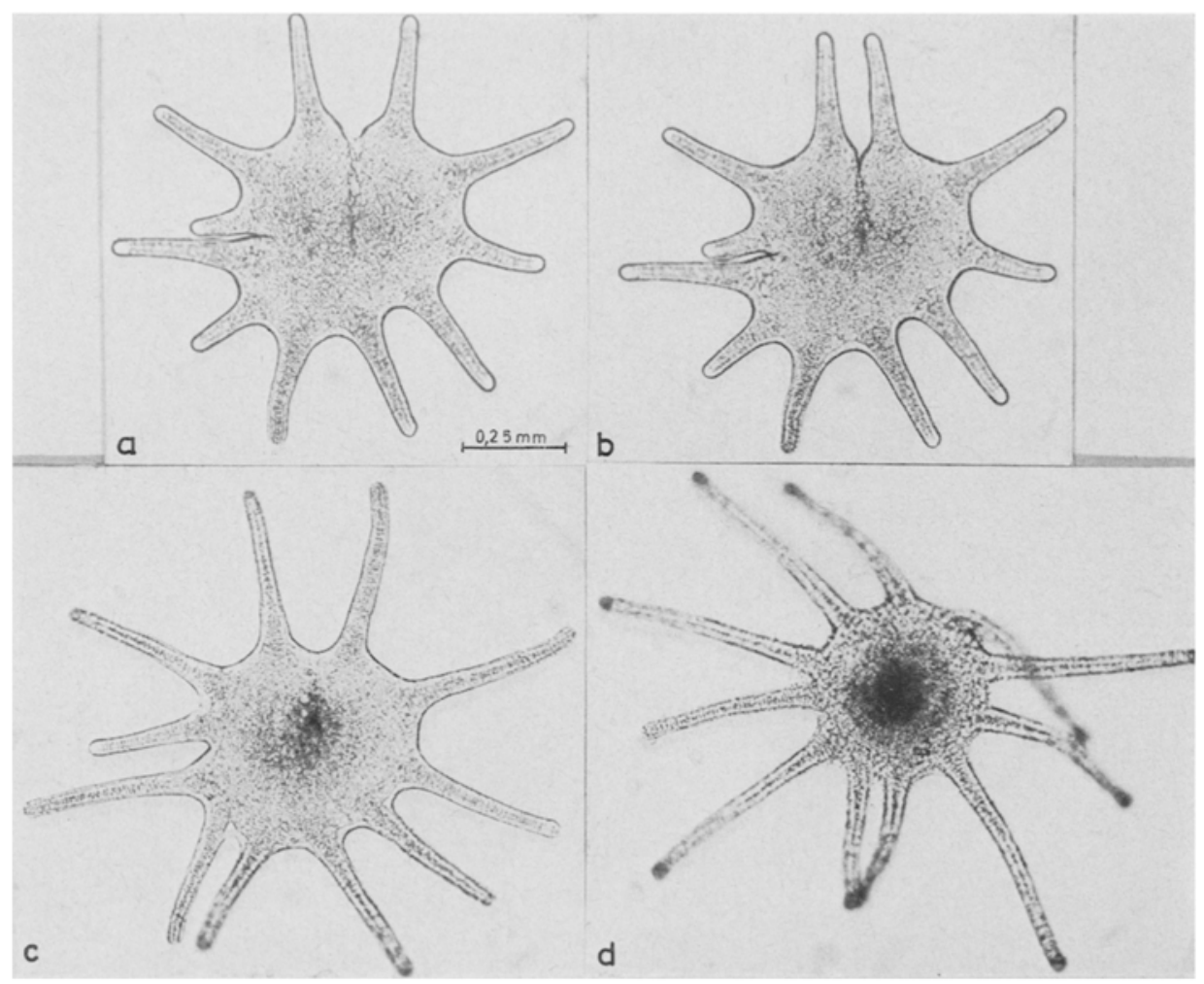

Abb. 1: Ectopleura dumortieri. (a): „Sternchenstadium“ mit 11 Tentakeln; Einschnitt zwischen zwei Tentakeln bis über die Körpermitte $11 \mathrm{~min}$ vor (a). (b): 17 min später. (c): 2 Stunden 8 min nach (b); Schnittwunde völlig verheilt. (d): 2 Stunden 47 min nach (c); sehr agile Proactinula. (Aus Zeitrafferaufnahme)

vorhandenen - in diesem Falle 14 - soweit wie möglich entfernt werden (a). Die Teilbilder lassen die recht erheblichen Umlagerungen und Neudifferenzierungen des Zellmateriales erkennen. Völlig ungestört von der Amputation der 14 Tentakel und den dabei entstandenen 14 kleinen Schnittwunden, die bereits 7 Minuten nach der Operation kaum noch zu bemerken sind, geht das Vorwachsen der aboralen Anlage des späteren Polypenstieles vonstatten. 40 Minuten nach Entfernung der Tentakel ist an der Stielanlage bereits das Ektoderm deutlich abgesetzt. 66 Minuten nach Teilbild (a) sprossen die ersten neuentstandenen Tentakel hervor $(f)$, und $z$ war an der Basis der aboralen Anlage des Stieles (g, h). In (f) sind auch die ersten Vorwölbungen der Oraltentakel erkennbar; in Teilbild (g) sind 3 Orakeltentakel- "Knospen“ deutlich wahrzunehmen. 4 Stunden nach der Operation weist der "alte Anteil " (in den Teilbildern rechts) lebhafte, rhythmisch ablaufende Kontraktionen auf - im Gegensatz zum Verhalten der aboralen Stielanlage - die weitere Z.R.-Aufnahmen verhindern. 


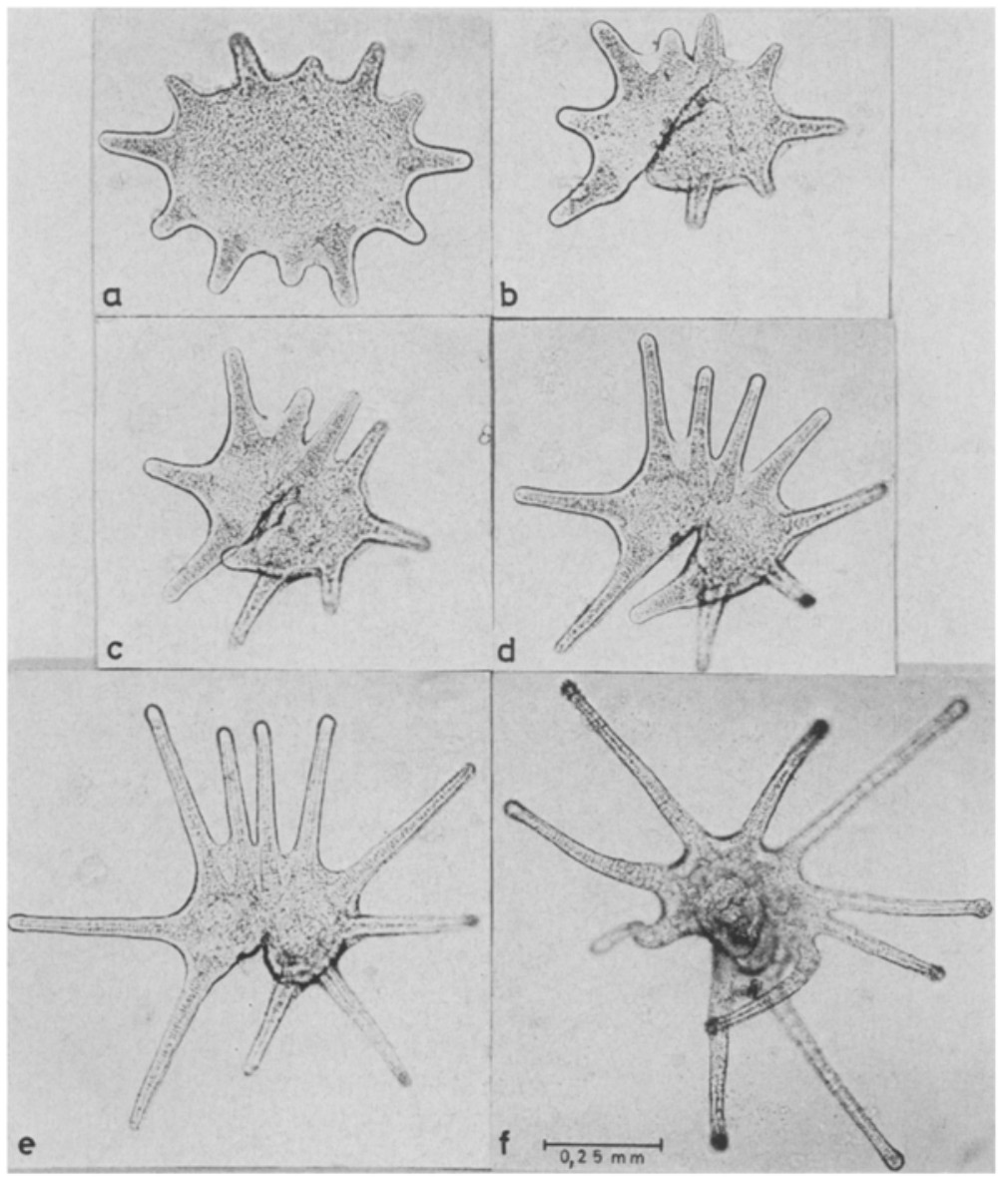

Abb. 2: Ectoplewra dumortieri. (a): "Sternchenstadium" mit 12 Tentakeln („disymmetrische" Anordnung). (b): 18 min nach (a); schräger Einschnitt bis weit über die Mitte des Körpers; 3 Tentakel zerstört. (c): 1 Stunde 6 min nach (b); neben der Schnittstelle wächst neuer Tentakel aus - in (b) als abgerundeter Stumpf erkennbar; Schnitt bis annähernd Körpermitte verheilt. (d): 1 Stunde 21 min nach (c); Heilung weiter fortgeschritten; neuer, 10. Tentakel stark gewachsen. (e): 2 Stunden 15 min nach (d); 10 lange Tentakel, noch gerade gestreckt; Schnittwunde nahezu geschlossen. (f): 3 Stunden 5 min nach (e); Schnittstelle nur noch als leichte Einbuchtung erkennbar; 10 sehr bewegliche Tentakel; aborale Stielanlage deutlich. (Mikroaufnahme nach dem lebenden Objekt)

Bei der "Total-Amputation" der 14 Tentakel dieser Proactinula wurde das Mikromesser möglichst an der Basis angesetzt. Es ist nicht auszuschließen, daß kleine Tentakelreste zurückgeblieben sind, zum mindesten bei einigen Fangarmen. Wenn diese Reste wieder auswachsen, liegt Regeneration vor und keine Neubildung im engeren Sinne. In Teilbild (h) erkennt man jedenfalls 5 wohlausgebildete Aboral-Tentakel und 3 (vielleicht 4) Orakeltentakel-Anlagen. Das Bewegungsverhalten ist als normal anzusprechen. 


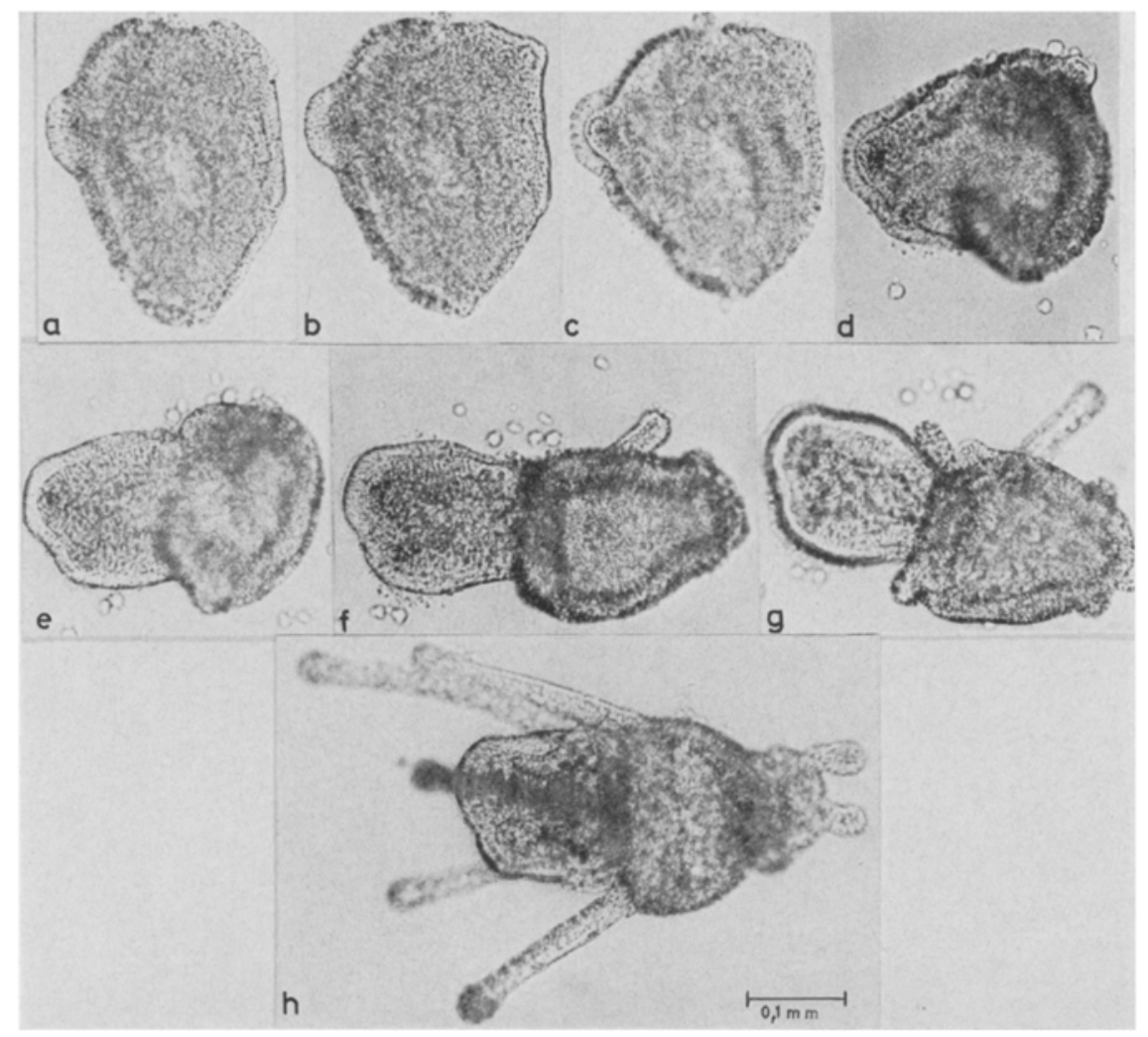

Abb. 3: Ectopleura dumortieri. Ein 12-14 Stunden altes "Sternchenstadium", welchem sämtliche 14 Tentakel entfernt wurden. (a): 7 min nach der Operation. (b): 33 min nach (a). (c): $33 \mathrm{~min}$ nach (b); Auswachsen der aboralen Stielanlage. (d): $56 \mathrm{~min}$ nad (c); Ektoderm des auswachsenden Stieles deutlich abgesetzt. (e): $66 \mathrm{~min}$. nach (d); Streckung und beginnende Differenzierung in Stiel- und Polypanlage. (f): 66 min nach (e); im Anlage-Gebiet des Polypen Auswachsen des ersten neuen 'Tentakels; Anlagen der Oraltentakel werden erkennbar. (g): 1 Stunde $58 \mathrm{~min}$ nach (f); 4 neue Tentakelanlagen; 3 Oraltentakel wachsen aus. (h): Etwa 8 Stunden seit Aufnahmebeginn; fast normale Actinula mit 7 aboralen ( 5 deutlich) und 4 Oraltentakeln; lebhafte Bewegungen verhindern weitere Zeitraffer-Aufnahmen. (Aus Zeitrafferaufnahme)

\section{Regeneration und Neubildung von Tentakeln der Proactinula}

Werden an einem "Sternchenstadium" mit 9 Tentakeln 4 operativ entfernt, so sind nach $31 / 4$ Stunden wieder 8 gut ausgebildete Fangarme erkennbar. Abbildung 4 zeigt einen derartigen Versuch in vier Teilbildern (a-d) einer Z.R.-Aufnahme. Eine Kontrollaufnahme 31 Stunden nach der Operation ergab eine sich normal verhaltende Actinula mit 8 aboralen und 3 oralen Tentakeln. Die Stielanlage ist in (d) deutlich zu erkennen. Auch hier ist eine Feststellung, ob eine wirkliche Neubildung oder eine Regeneration von kleinen Tentakelresten vorliegt, nicht einwandfrei möglich. 


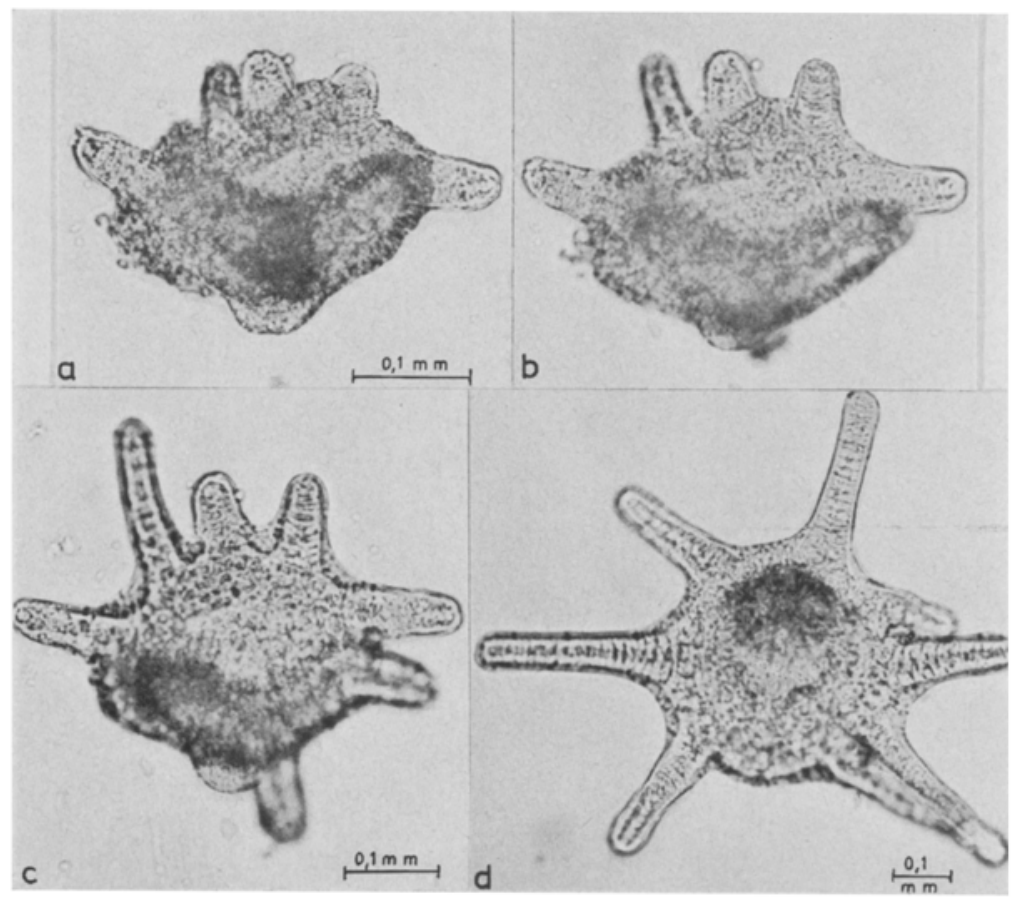

Abb. 4: Ectopleura dumortieri. Alteres "Sternchenstadium" mit 9 Tentakeln. 4 Tentakel wurden entfernt. Stielanlage deutlich. (a): 10 min nach der Operation. (b): 20 min $36 \mathrm{sec}$ nach (a); die restlichen 5 Tentakel sind gewachsen. (c): 56 min nach (b); Verlängerung der 5 nach der Operation verbliebenen Tentakel; 2 neue Tentakel. (d): 1 Stunde 47 min nach (c) (3 Stunden 12 min nach Aufnahmebeginn); 8 Tentakel; Stielanlage als dunkle Region erkennbar; Objekt hat sich gedreht und wird jetzt von der Fläche gesehen. (A us Zeitrafferfilmaufnahme)

\section{Völlige Durchtrennung eines beginnenden "Sternchen-Stadium"}

Bei dem in Abbildung 5 (a-e) dargestellten Versuch wurde ein erst kurze Tentakelanlagen aufweisendes junges "Sternchen-Stadium" nicht genau in der Mitte durchschnitten. Das große Teilstück ( $a: 1)$ zeigt an der Schnittstelle etwa 7 bis 8 aus dem Gewebeverband losgerissene Zellen ( 7 Minuten nach der Operation). Der abgetrennte kleinere Anteil (2) hat bereits die Schnittfläche eingekrümmt: „Abkugelungstendenz“.

Versuche an frühen „Sternchen-Stadien" haben den Vorteil, daß noch keine Tentakelbewegungen erfolgen. Regenerations- und Neubildungsvorgänge können im Z.R.-Laufbild ohne störende Kontraktionen und Ortsverlagerungen beobachtet werden. Bald einsetzende Bewegungen lassen jedoch auch hier eine Teilbild-Analyse wenig aussichtsreich erscheinen.

In Teilbild (e) (6 Stunden nach der Operation) liegen die beiden Teilstücke 1 und 2 noch dicht nebeneinander. 3 Stunden und 22 Minuten nach der Trennung 


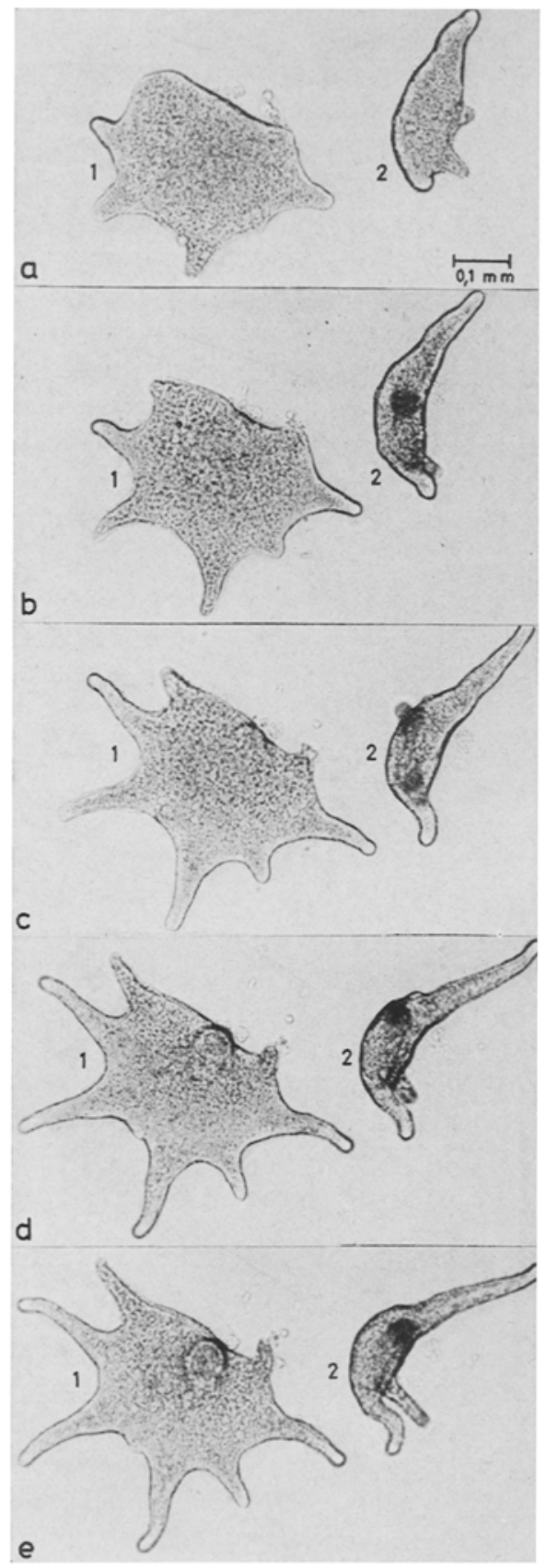




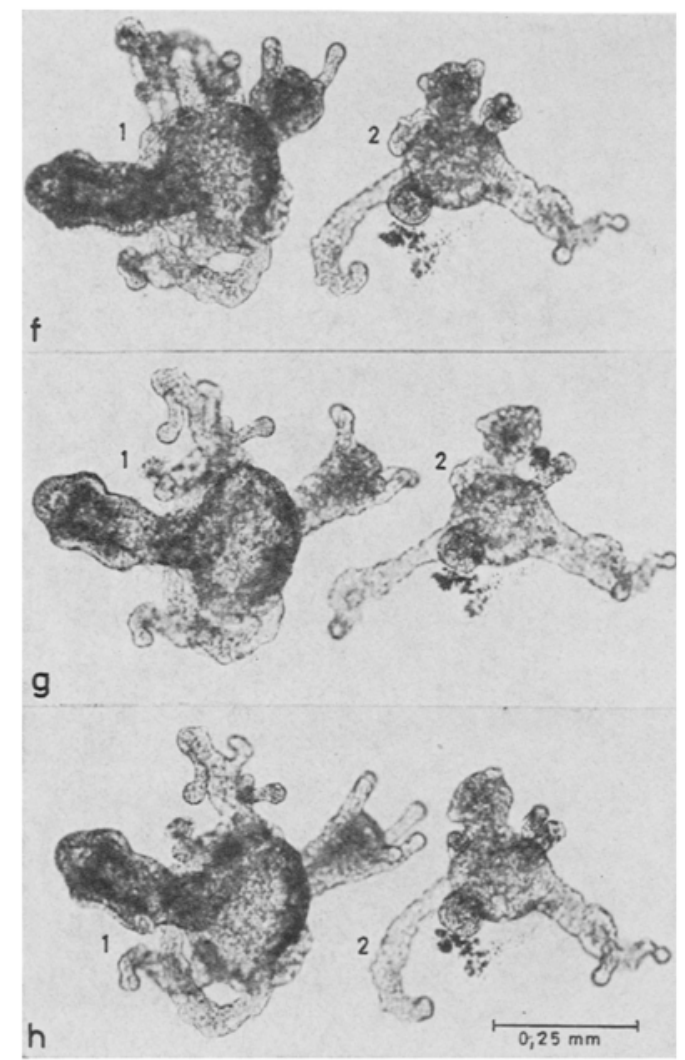

Abb. 5: Ectopleura dumortieri. Beginnendes "Sternchenstadium" wurde in größeren (1) und kleineren Anteil (2) getrennt. (a): 50 min nach der Operation; der größere Anteil 1 hat 4 deutliche und 3 angedeutete Tentakelanlagen, der kleinere Anteil 2 vier. (b): 1 Stunde 55 min nach (a); in 1 sind 4 Tentakel gewachsen; die Anlagen der 3 weiteren Tentakel deutlicher vorgewölbt; 2 ist länger geworden. (c): 1 Stunde 20 min nach (b); Teilstüdk 1 läßt beginnende Stielanlage erkennen; Tentakel sind weiter ausgewachsen; das eine Ende von 2 wächst zu einem langen Tentakel aus; (d): 1 Stunde 20 min nach (c); Teilstück 1 hat 6 lange und 1 kurzen Tentakel; Stielanlage stärker vorgewölbt. 2 zeigt außer dem langen zwei kurze Tentakel am entgegengesetzten Ende. (e): $40 \mathrm{~min}$ nach (d). Teilstück 1 hat 6 typische Tentakel nebst einem. nur langsam vorwachsenden Tentakel dicht bei der Schnittstelle; dunkle Region in 2 nicht sicher zu deuten; seit der Operation sind 6 Stunden vergangen. $(f-h):$ Der größere (1) und kleinere (2) Anteil der Regenerationsphasen (a-e) 15 Stunden nach (e); 3 Bewegungsphasen der sehr agilen, jedoch offenbar nicht normal weiterentwickelten Teilstücke 1 und 2; Teilstück 1 zeigt deutliche, bereits gestreckte Stielanlage (links); rechts 4 Oraltentakel; Anzahl der aboralen Tentakel infolge horer "Verkrampfung nicht einwandfrei feststellbar; sie sind in lebhatter Bewegung, jedoch nicht zur Streckung fähig; 2: „Stielanlage“? (links unten) erkennbar; gegenüber 2 kleine Oraltentakel. „Verkrampte“ Bewegung wie bei 1. (Aus Zeitrafferaufnahme)

(Abb. 5c) läßt 1 eine deutliche kleine Anlage des aboralen Stieles erkennen; sie liegt unverändert im ursprünglichen Zentrum des "Sternchens". 4 Stunden und 42 Minuten nach der Durchschneidung (e) ragt die aborale Stielanlage erheblich über die Körperoberfläche empor. Neben ihr ist ein kleiner Tentakel aus der bereits in (a) erkennbaren ursprünglichen Anlage an der Grenze der Schnittwunde ausgewachsen. 
Einwandfreie Neubildungen von Fangarmen sind in diesem Versuch nicht festzustellen. Beachtlich ist die weitgehende Verlagerung des Zellmaterials in die bereits vor der Amputation vorhandenen Tentakelanlagen des kleinen Teilstïckes 2; das ganze Teilstiuck geht in drei Tentakel auf.

Die beiden Teilstücke dieses Zerschneidungsversuches wiesen 15 Stunden nach der Phase des Teilbildes (e) (Abb. 5), also 20 Stunden nach der Operation, das Aussehen der Teilbilder $(f-h)$ auf. Unter Z.R. ergab sich ein lebhaftes, unkoordiniertes Bewegungsverhalten. Die lang ausgewachsenen Tentakel erscheinen verkrampt und sind unfähig, sich in normaler Weise zu strecken. Im großen Teilstück 1 ist die aborale Anlage (links) bereits „stielförmig" ausgewachsen und zu Kontraktionen fähig. Die Oralseite (rechts) zeigt jetzt vier kurze Oraltentakel, die von einem relativ langen Körperfortsatz ausgehen, was als nicht normal anzusehen ist. Der kleine Anteil (2) verhält sich ähnlich. Die zwischen den drei verkrampften Tentakeln entstandenen kleinen Fortsätze, von denen einer abgestorbenes Zellmaterial ausstößt, können morphologisch nicht einwandfrei gedeutet werden. Die Weiterentwidklung in $(f-h)$ hat pathologischen Charakter angenommen. Auch 2 bewegt sich unter Z.R. lebhaft auf der Stelle.

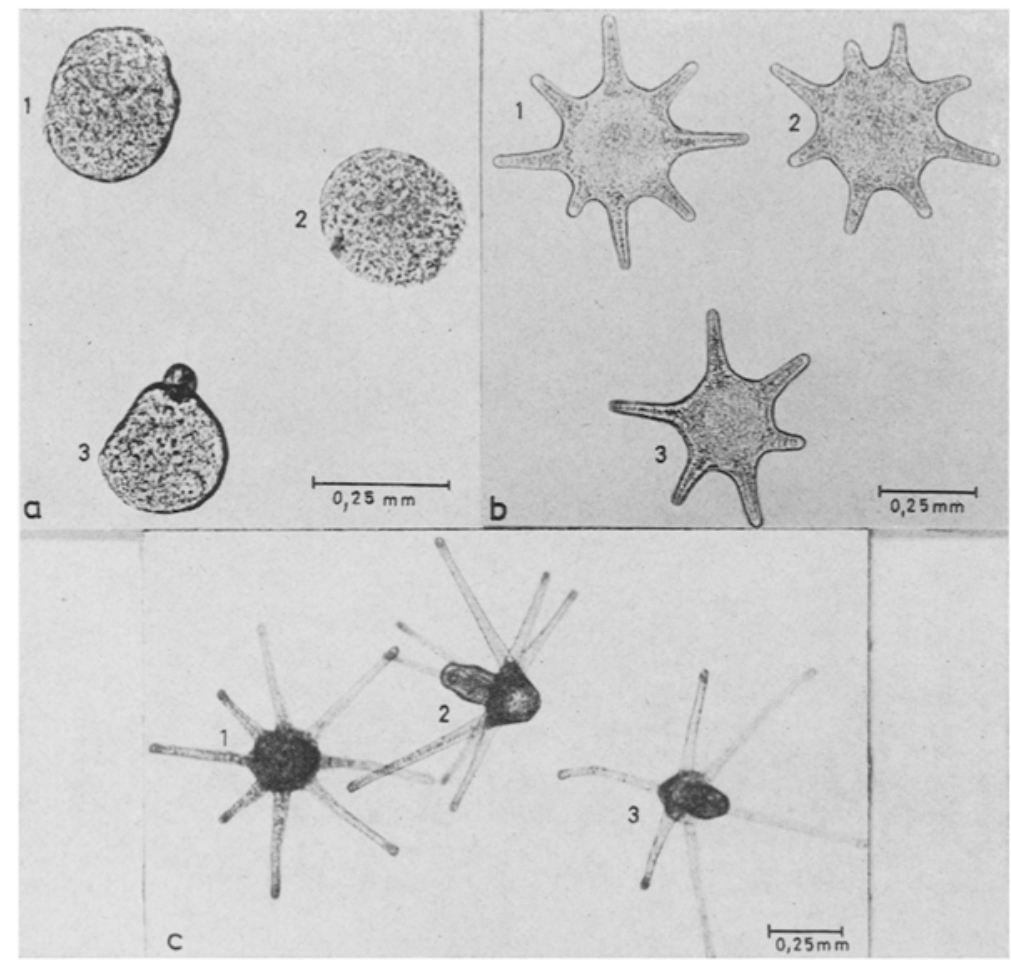

Abb. 6: Ectopleura dumortieri. 256-Blastomerenstadium wurde in 3 fast gleiche Teile zerschnitten. (a): $17 \mathrm{~min}$. nach der Operation; 3 stößt Blastomere ab. (b): 12 Stunden $28 \mathrm{~min}$ nach (a); 3 wohlausgebildete "Sternchen"; 1 und 2 mit je 8, 3 mit 6 Tentakeln; in der Mitte yon 1: Anlage des Stieles erkennbar; bei 2 erste zentrale Blastomerenanhäufung. (c): 7 Stunden 4 min nach (b). 1 und 2: Actinulae mit je 8, 3: mit 6 Tentakeln; die drei "Regenerate" haben insgesamt 22 Tentakel gebildet. (Mikroaufnahmen nach dem lebenden Objekt) 


\section{Zerschneidungsversuche in mehrere bis viele Teilstücke}

Wie bereits betont, ist nach Amputationen der Tentakel die Entscheidung oft nicht sicher, ob Regenerate oder wirkliche Neubildungen vorliegen. Klarheit ist nur $\mathrm{zu}$ erreichen, wenn die mikrochirurgischen Eingriffe an frühen Entwicklungsstadien vorgenommen werden, also noch keine Andeutungen von Tentakelanlagen erkennbar sind. In den folgenden Versuchsreihen werden die Ausgangsstadien in eine immer gröBere Zahl von Teilstücken zerlegt, maximal bis 10 .

Abbildung 6 geht von einem etwa 256-Blastomerenstadium aus; dieses wird in drei nahezu gleich große Teile zerschnitten (a). Die drei 12 Stunden und 28 Minuten nach (a) vorliegenden Proactinulae (b), die einen normalen Habitus aufweisen und sich auch normal verhalten, weisen zusammen 22 Tentakel auf, also doppelt so viel wie normalerweise gebildet werden (AURICH 1958). 7 Stunden später (c) liegen drei

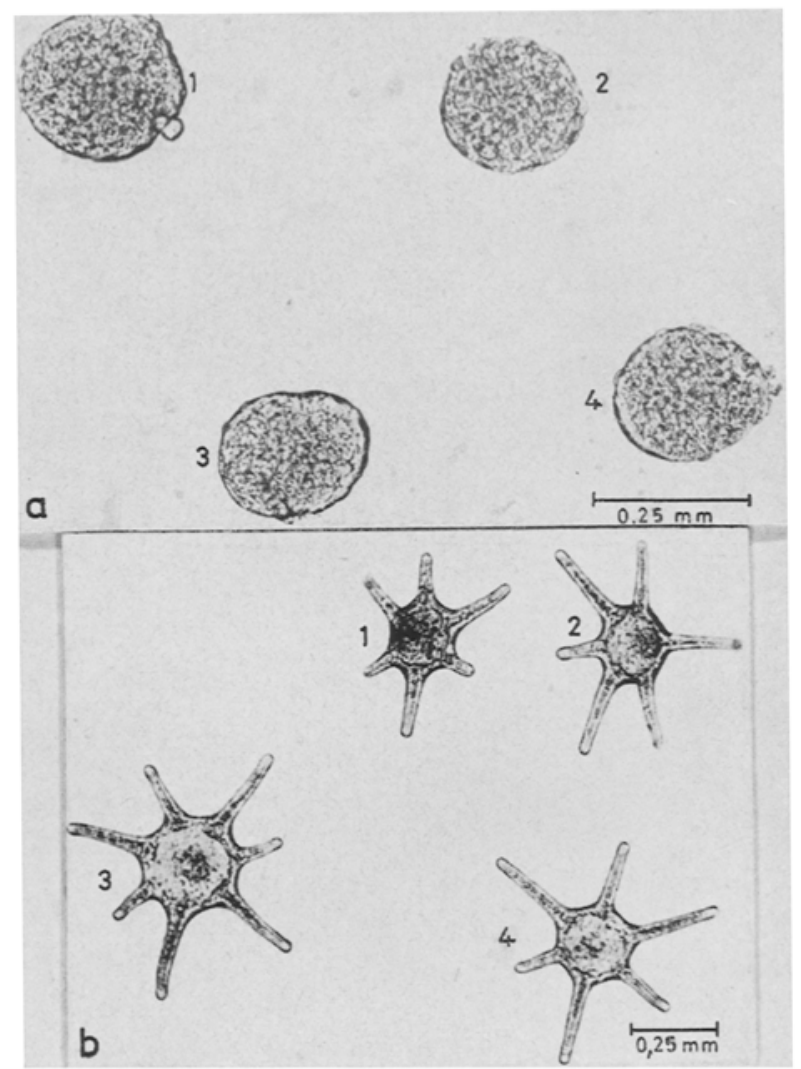

Abb. 7: Ectoplewra dumortieri. Ein langgestrecktes Entwicklungsstadium wurde etwa 1 Stunde 30 min nach Furchungsbeginn in 4 nahezu gleiche Teile zerschnitten (1-4). (a): 43 min nach der Operation; 1,3 und 4 stoßen verletzte Blastomere ab; völlige Abkugelung. (b): 11 Stunden 54 min nach (a); aus den vier Teilstücken wurden 4 "Sternchen" gebildet: 1,2 und 4 mit je 6, 3 mit 7 Tentakeln; Stielanlagen angedeutet; es sind insgesamt 25 Tentakel von den 4 Teilstücken gebildet worden. (Mikroaufnahmen nach dem lebenden Objekt) 


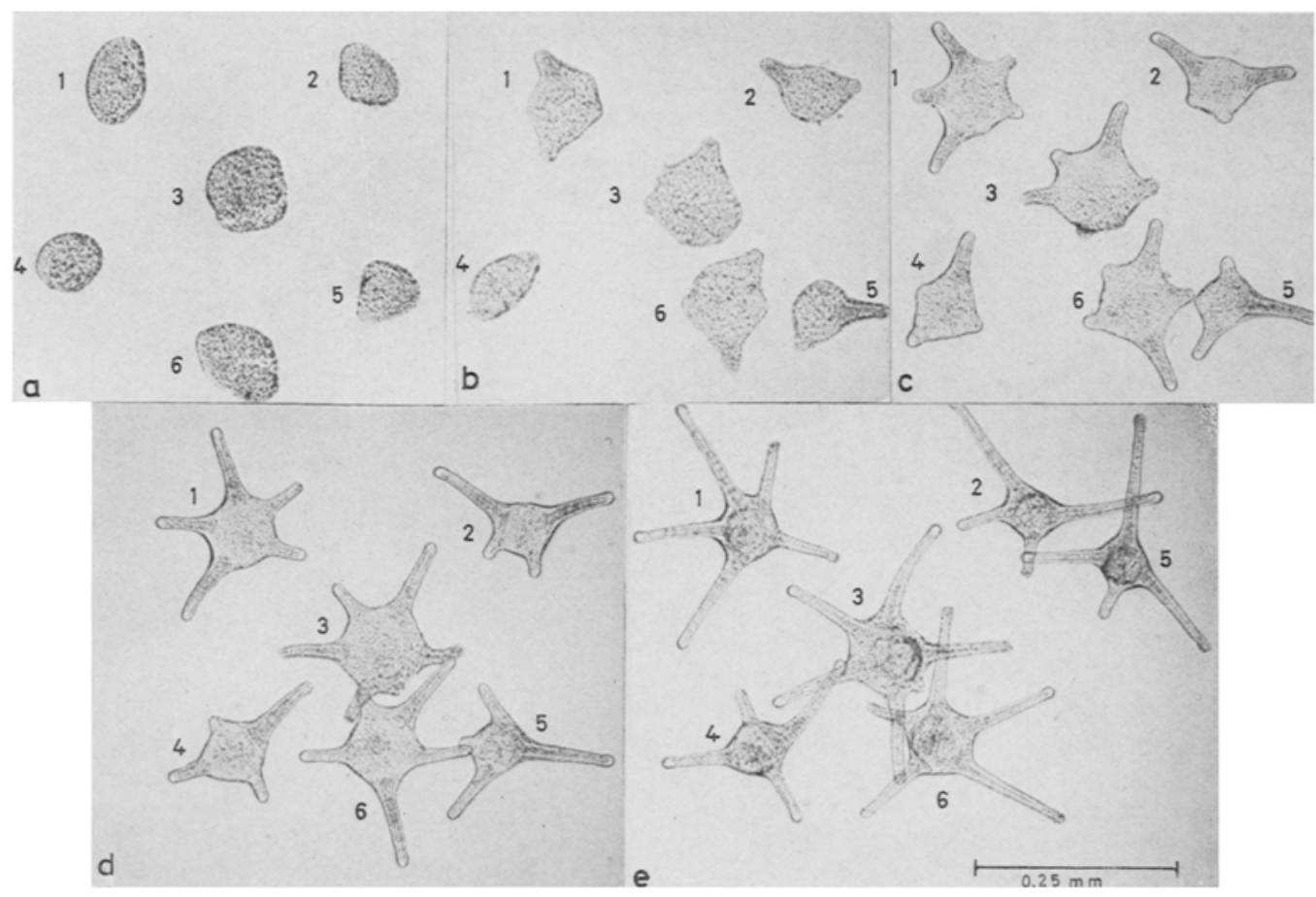

Abb. 8: Ectopleura dumortieri. Ein etwa 3-4 Stunden altes beginnendes "Sternchenstadium“ mit 6 Tentakelanlagen wurde in 6 Teile zerschnitten. (a): $5 \mathrm{~min}$ nach der Operation; Teilstüdke verschieden groß. (b): 1 Stunde $6 \mathrm{~min} 36 \mathrm{sec}$ nach (a); sämtliche 6 Teilstucke weisen Tentakelanlagen auf. (c): 2 Stunden 13 min 18 sec nach (b); Tentakel weiter vorgewachsen; Gesamtzahl der Tentakelanlagen der Teilstücke 1-6:27. (d): 2 Stunden $13 \mathrm{~min} 18 \mathrm{sec}$ nach (c); es liegen 6 kleine "Sternchen" vor; außer beim größten Teilstück 3 mit einer 6 . kleinen Tentakelanlage, keine weiteren neuen Tentakel angelegt. (e): 3 Stunden $20 \mathrm{~min}$ nach (d); Tentakel erheblich verlängert; in sämtlichen 6 "Sternchen“, die Bewegungen und Ortsverlagerungen aufweisen, ist die Anlage des Stiels erkennbar; seit der Operation sind 8 Stunden 58 min vergangen. (Aus Zeitrafferaufnahme)

normale Proactinulae vor mit deutlicher Stielanlage (2 und 3). Die Anzahl der Tentakel ist nicht weiter vergrößert worden. In Abbildung 6 (c) läßt 2 besonders deutlich das normale "Einschlagen" der 8 Tentakel in Richtung auf die aborale Stielanlage erkennen.

Nach dem 8. Teilungsschnitt des Ectopleura-Eies ist also die Anzahl der Tentakel des späteren "Sternchen-Stadium", der Proactinula, noch nicht endgültig festgelegt: drei Teilstücke können doppelt so viele Tentakel ausbilden wie eine Proactinula normalerweise aufweist.

Wenn mehr Teilstücke mikrochirurgisch erhalten werden, nimmt die Anzahl der neugebildeten Tentakel nicht in strenger Korrelation zu. Abbildung 7 (a) (43 Minuten nach der Zerteilung eines $1 \frac{1}{2}$ Stunden alten Entwicklungsstadium) zeigt vier abgekugelte Teilstücke ungefähr gleicher Größe. Die vier nach fast 12 Stunden vorliegenden "Sternchen" (b) mit deutlicher aboraler Stielanlage haben zusammen 25 wohlausgebildete Tentakel, also 13 bis 14 mehr als bei ungestörter Entwicklung maximal 

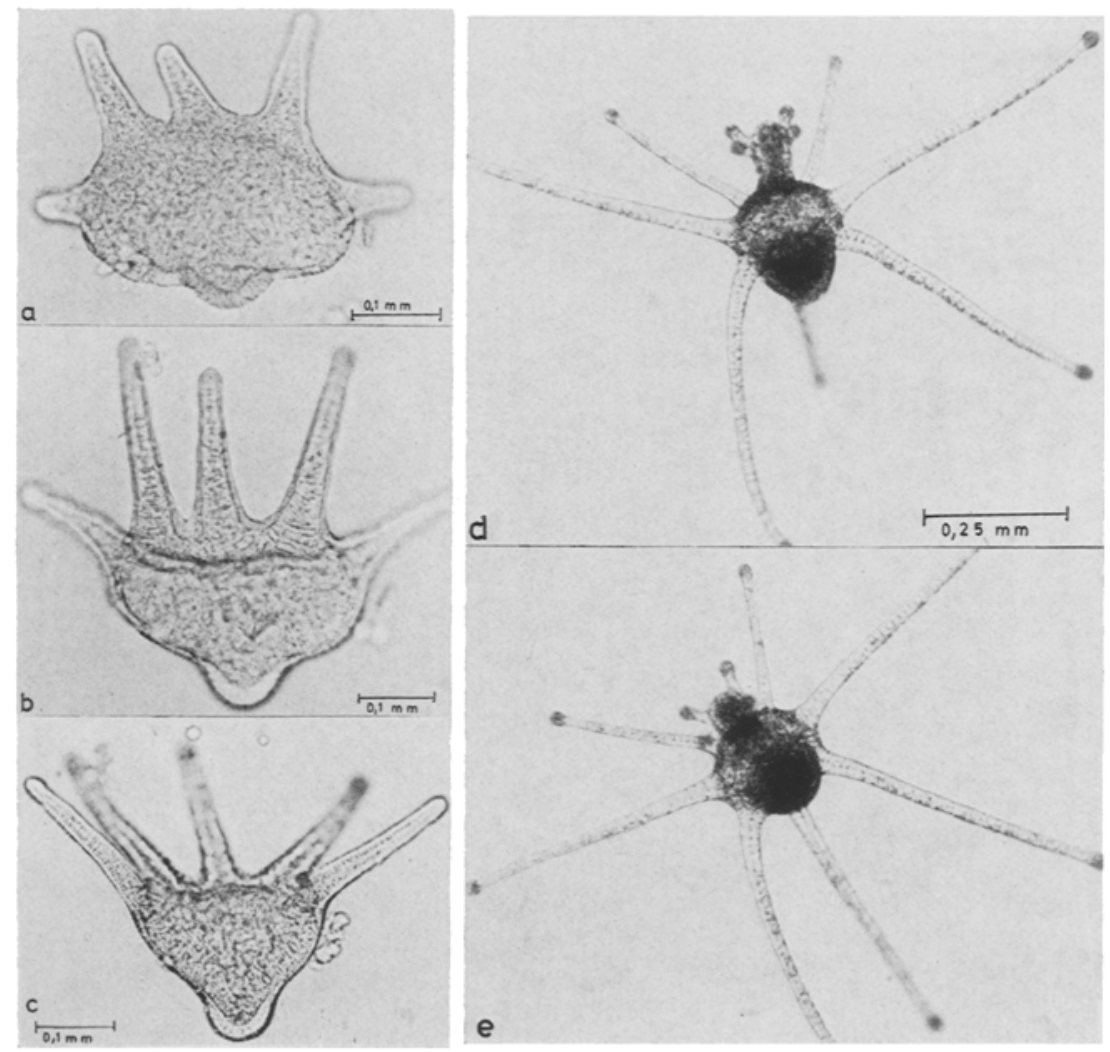

Abb. 9 a-e: Ectopletra dumortieri. Ein "Sternchenstadium" mit 10 Tentakeln wurde in der Mitte zerschnitten. (a): 18 min nach der Durchtrennung; an der Schnittfläche wölbt sich Stielanlage vor; Ektoderm deutlich abgesetzt. (b): 1 Stunde nach (a); die von der Operation nicht betroffenen 5 Tentakel stark gestreckt; Stielanlage weiter vorgewachsen; Körperform actinulaähnlicher. (c): 1 Stunde 42 min nach (b); lebhafte Tentakelbewegungen; Stielanlage verlängert. (d): 2 Tage 3 Stunden 9 min nach (a); wohlausgebildete Actinula; 2 Tentakel neu gebildet; 4 Oraltentakel. (e): 1,5 min nach (d); Körper der Actinula kontrahiert; Oraltentakel gestreckt.

gebildet worden wären, soweit die Variabilität der Tentakel bei der Proactinula bisher bekannt ist. Das aus dem Teilstück 3 hervorgegangene "Sternchen " weist einen Tentakel mehr auf (7) gegenüber 6 bei 1,2 und 4 .

Bei dem in Abbildung 8 dargestellten Versuch wurden 6 verschieden große Teilstücke mit drei Schnitten erzielt. Der etwa 3 bis 4 Stunden alte Keim zeigt 6 kleine Vorwölbungen, erste Tentakelanlagen. Jede Anlage wurde durch die Schnittführung möglichst genau halbiert. 8 Stunden und 58 Minuten nach dem Zerschneiden des Keimes ist die Summe aller Tentakel der 6 unter Z.R. ihre Beweglichkeit zeigenden "Sternchen “ 24, variierend zwischen 6 und 4 in deutlicher Abhängigkeit von der zufälligen Größe des Teilstücks. Selbst die kleineren Anteile, mit nur vier Tentakeln, zeigen deutliche aborale Stielanlagen.

In Abbildung 9 wurde ein 10 Tentakel aufweisendes "Sternchen-Stadium" in ein 
großes, etwa die Hälfte des Körpers enthaltendes Teilstück und 2 kleine Anteile zerschnitten. Aus dem großen Teil ist nach 2 Tagen und 3 Stunden eine, bis auf die ge-

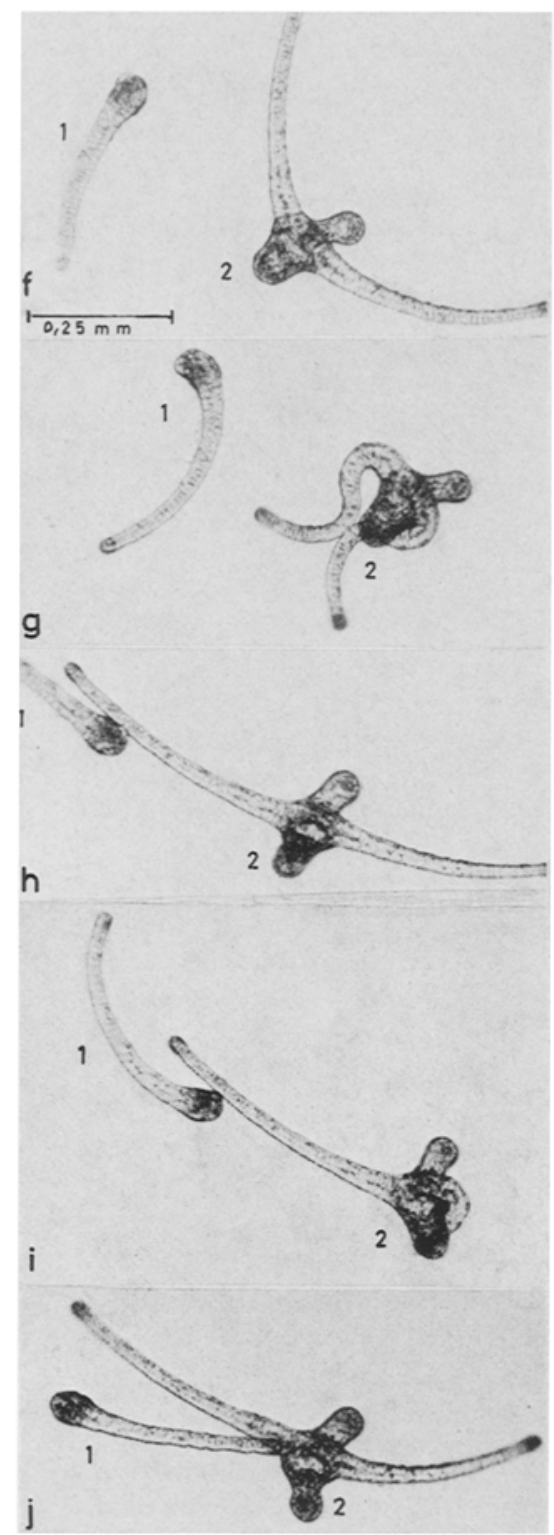

Abb. $9 \mathrm{f}$-j: Ectopleura dumortieri. Die beiden kleineren bei der Operation entstandenen Teilstücke $(1,2)$ in ihrem Bewegungsverhalten in 5 Phasen. (g): 20 sec nach (f). (h): $40 \mathrm{sec}$ nach $(\mathrm{g})$. (i): $20 \mathrm{sec}$ nach (h). (j): 20 sec nach (i); Teilstück 1 ist zu einem "Tentakel" ausgewachsen, der sich beugen und strecken kann ( $\mathrm{i}-\mathrm{j})$; Teilstück 2 hat 2 Tentakel, eine Stielanlage sowie die Basis für die Oraltentakel ausgebildet; die beiden langen Tentakel sind sehr beweglich und vermögen sich völlig einzukrümmen $(\mathrm{g}, \mathrm{i} ; 2)$. (Aus Zeitrafferaufnahme) 
ringere Tentakelzahl, normale Actinula geworden, die zwei neue Tentakel gebildet hat (d) und unter Z.R. lebhafte Bewegungen ausführt (e).

Von den beiden kleinen Anteilen $(f-j)$ besteht 1 nur aus einem ausgewachsenen Tentakel; die große Agilität geht aus den Teilbildern $\mathrm{f}-\mathrm{j}$ deutlich hervor. Nicht minder beweglich ist das Teilstück 2, das sich aus den Anlagen des aboralen Stiels, der Oraltentakel-Anlage und zwei sehr kontraktilen Tentakeln zusammensetzt.

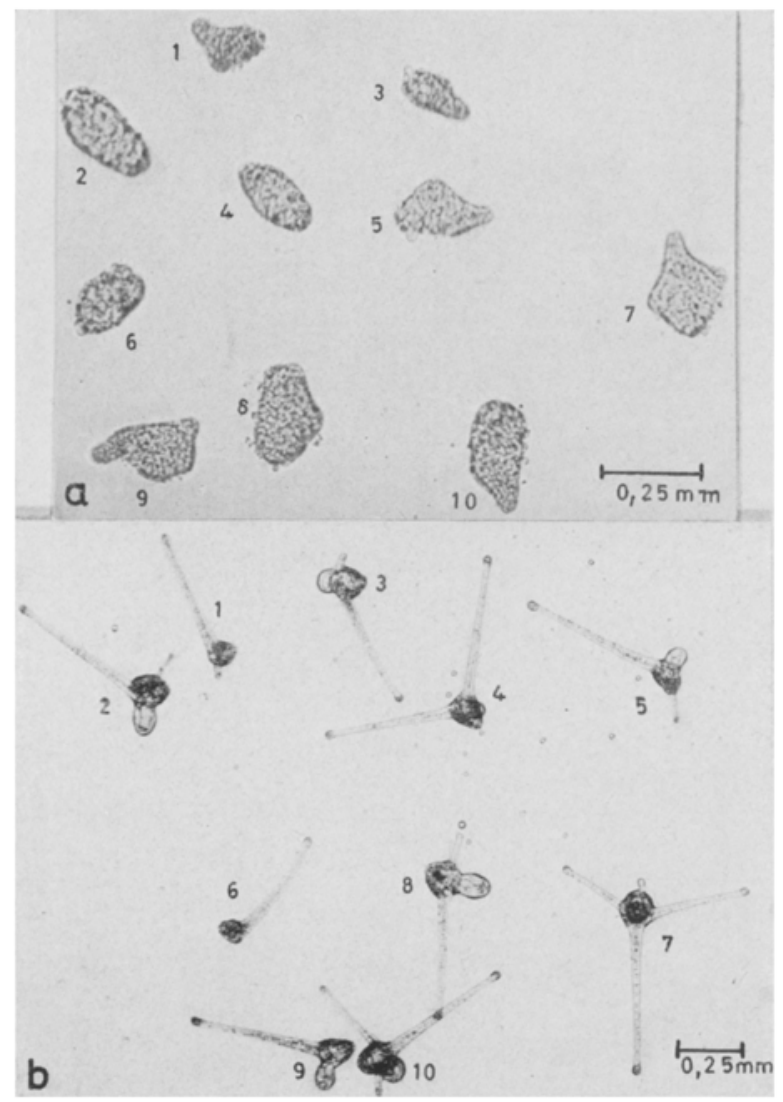

Abb, 10: Ectoplesura dumortieri. (a): Ein 91/2 Stunden altes "Sternchenstadium ${ }^{c}$ mit 11 Tentakelanlagen wurde in 10 Teilstücke zerschnitten (ein Schnitt in der "Längsachse", vier Schnitte in Querrichtung). (b): 131/2 Stunden nach (a); sätntliche 10 Teilstücke sind ausgewachsen; insgesamt sind 16 langgestreckte Tentakel und 6 Tentakelanlagen ausgebildet worden; mindestens

6 Teilstücke weisen eine Stielanlage auf. (Mikroaufnahmen nach dem lebenden Objekt)

Gegenüber dem großen Anteil (a-e) ist die Beweglichkeit der beiden kleinen Gebilde erheblich größer, was bei dem kleinen „Volumen“ (1) erstaunlich ist. Die große Agilität verhindert die Teilbild-Analyse des regenerativen Wachstums von 1 und $2(\mathrm{f}-\mathrm{j})$.

Sogar die Zerteilung eines 11 Tentakel aufweisenden. „Sternchens" in 10 Teile (Abb. 10a, b) ergab ein Auswachsen sämtlicher Teilstiicke, auch der kleinsten $(1,3)$. 
In (b) liegen, 13/2 Stunden nach (a) insgesamt 22 Tentakel vor, wobei die noch kurzen, im Auswachsen befindlichen, mitgerechnet sind. Es sind also 11 Tentakel mit Sicherheit neugebildet worden. Das Ausgangssternchen hätte außer den bereits vorhandenen 11 keine neuen Fangarme mehr angelegt.

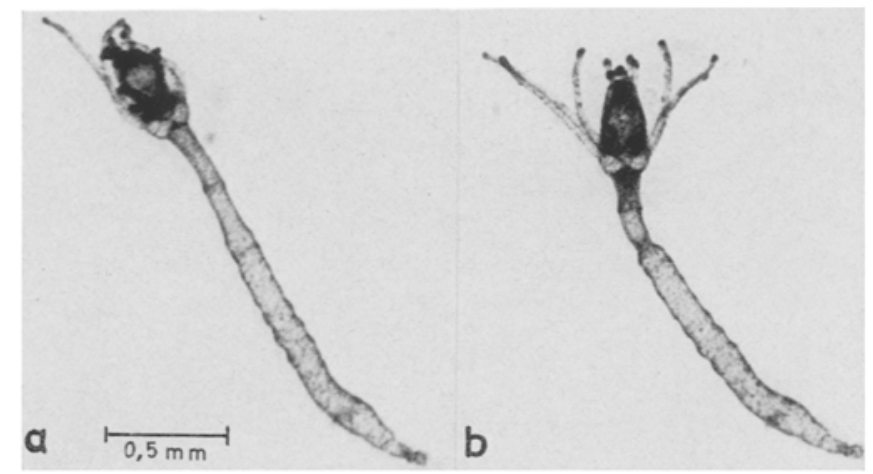

Abb. 11: Ectopleura dumortieri. 9 Tage alter Polyp. (a): Stiel gestredkt, Tentakel eingeschlagen. (b): Stiel kontrahiert, Tentakel in "Fangstellung". Der Polyp ist aus 9 Tage vorher in 4 Stücke zerschnittenem "Sternchenstadium" mit 11 Tentakeln hervorgegangen, und zwar aus einem der beiden großen Teilstücke mit 5 Tentakeln. Völlig normaler Polyp, der Nahrung aufnimmt. (Aus Zeitrafferaufnahme)

Daß aus kleinen, mikrochirurgisch gewonnenen Teilstücken von "Sternchen-Stadien" normale Ectopleura-Polypen entstehen können, die die typischen Fangbewegungen mit ihren Tentakeln ausführen und zum Beutefang befähigt sind, beweist Abbildung $11(\mathrm{a}, \mathrm{b})$. Der 9 Tage alte Polyp unterscheidet sich lediglich durch die geringere Tentakelzahl vom normalen Polypen.

\section{BESPRECHUNG DER ERGEBNISSE}

Mikrochirurgische Untersuchungen an der sternchenförmigen Proactinula von Ectopleura dumortieri ergaben ein sehr großes Wundheilungs- und Regenerationsvermögen. Dies wird aus der Tatsache verständlich, daß aus dem in der Fläche ausgebreiteten rundlichen "Sternchen" randlich die radiär angeordneten Tentakel gebildet werden, aus der zentralen Region der aborale Stiel des Polypen auswächst und auf der entgegengesetzten Seite die Oraltentakel und die Mundregion entstehen, also ein relativ einfaches, direktes ontogenetisches „Verfahren“ vorliegt.

Klaffende Schnitte in das scheibenförmige "Sternchen" schließen sich in wenigen Minuten und verheilen nach etwa 2 Stunden völlig. Die Proactinula übersteht auch die Entfernung sämtlicher Tentakel (maximal sind 14 vorhanden); es entwickelt sich trotz der zahlreichen kleinen peripheren Schnittverletzungen an der Basis der Tentakel eine Actinula, jedoch mit einer geringeren Anzahl "regenerierter" Tentakel, sowohl aboraler wie oraler. 
Es muß scharf unterschieden werden zwischen regenerierten und neugebildeten Tentakeln, da es nicht möglich ist, nach der Operation einwandfrei festzustellen, ob wirklich der Tentakel an seiner Basis völlig abgetrennt wurde - sofern dies technisch überhaupt möglich ist, ohne eine größere Verletzung des Ektoderm an der Peripherie hervorzurufen.

Zur Untersuchung der Frage, ob wirkliche Neubildungen von Fangarmen nach mehr oder minder weitgehenden mikrochirurgischen Eingriffen an der Proactinula möglich sind, müssen frühere Entwicklungsstadien für die Versuche verwandt werden, die noch keine über die Peripherie hinausragenden Tentakelanlagen aufweisen. Außerdem sind diese frühen Versuchsobjekte total in eine verschieden große Anzahl von Teilstücken zu zerschneiden. Aus deren Verhalten nach der Operation lassen sich dann Schlïsse ziehen, ob Neubildungen möglich sind. Einschränkend ist zu sagen, daß diese Folgerungen nur aus der Gesamtzahl der bei den Teilstiicken am Versuchsende vorliegenden Fangarme gezogen werden können. Beim einzelnen Tentakel kann nicht festgestellt werden, ob er ein Regenerat oder eine Neubildung ist.

Da bei den totalen Zerschneidungsversuchen - im Maximum lagen 10 Teilstücke vor - oft mehr als die doppelte Anzahl von Tentakeln (bei der Addition der Fangarme sämtlicher Teilstücke des Versuchs) vorlag, als ein "Sternchen " normalerweise zu bilden vermag, so ist die Frage mit Sicherheit zu bejahen, daß nach Verletzungen der Proactinula neue Fangarme gebildet werden können, und zwar an Stellen, an denen im Normalfall bestimmt keine Tentakel gebildet worden wären.

Die Anzahl der an Proactinula-Teilstücken gebildeten Tentakel ist in gewissem Umfang von der Größe des Fragments abhängig; sehr kleine Teile können in der Bildung eines Tentakels völlig aufgehen und trotz der geringen Größe sehr beweglich sein. Bemerkenswert ist, daß bei sämtlichen Total-Zerschneidungsversuchen jedes Teilstück Tentakel und meist sogar aborale Stielanlagen und Oraltentakel-Anlagen ausbildete.

Z.R.-Aufnahmen erwiesen sich als notwendig zur Nachprüfung der Vitalität und des Bewegungsverhaltens der Fragmente.

Die Regenerationsfähigkeit von Ectopleura geht so weit, daß beispielsweise aus dem vierten Teil eines 11 Tentakel aufweisenden "Sternchenstadium“ nach 9 Tagen ein normaler Polyp mit 6 aboralen und 5 oralen Tentakeln gebildet wird.

Eine genaue „Einengung“ des Zeitpunktes der ersten Anlage der Tentakel, bevor diese als kleine Vorwölbungen in der Randzone sichtbar werden, erfordert wesentlich umfangreichere Versuchsreihen als das vorhandene Eimaterial und die zur Verfügung stehende Zeit vorzunehmen gestattete.

\section{ZUSAMMENFASSUNG}

1. Entwicklungsstadien von Ectopleura dumortieri van Beneden (Tubularidae, Athecatae-Anthomedusae) vom 8. Teilungsschritt (256 Blastomere) an bis zur Proactinula ("Sternchen-Stadium“) mit bereits beweglichen Tentakeln wurden mikrochirurgischen Eingriffen ausgesetzt. 
2. Einschnitte in den Körper der Proactinula zwischen zwei Tentakeln heilen in zwei Stunden zu, ohne Spuren der Verletzung zu hinterlassen.

3. Trotz Amputation sämtlicher Tentakel eines „Sternchens" entsteht eine sich normal verhaltende Proactinula, jedoch mit reduzierter Tentakelzahl.

4. Völlige Durchtrennung eines "Sternchens" wird gut vertragen. In jedem Fall bilden die Teilstücke Tentakel aus, die sich meist normal strecken und bewegen.

5. Ob Regeneration oder Neubildung von Fangarmen nach Verletzungen vorliegt, läßt sich nur durch Versuche an frühen Entwicklungsstadien entscheiden, und zwar nicht für den einzelnen Tentakel, sondern nur an Hand der Summe aller Tentakel sämtlicher Teilstücke; diese beträgt oft mehr als das Doppelte der Maximalzahl, welche eine normale Proactinula zu bilden vermag.

6. Aus allen 10 Teilstücken eines "Sternchens" entstanden kleine "Proactinulae" mit 3 bis 4 normalen Tentakeln, zum Teil deutlich erkennbarer aboraler Stielanlage und vorwachsender Basis der Oraltentakel.

7. Aus nicht zu kleinen Teilstücken können ganze Polypen entstehen, die sich mit langem Stiel festsetzen; sie sind zum Beutefang befähigt.

8. Die Vitalität der Regenerate und Neubildungen sowie ihr Bewegungsverhalten werden durch Zeitraffer-Filmaufnahmen kontrolliert.

\section{ZITIERTE LITERATUR}

Aurich, H, J., 1958, Verbreitung der Medusen und Actinulae von Ectoplewra dumortieri (VAN BENEDEN) und Hybocodon prolifer L. Agassiz in der südlichen Nordsee. Helgoländer wiss. Meeresunters. 6, 207-228.

Aurich, H. \& Werner, B., 1955. Uber die Entwicklung des Polypen von Ectopleura dumortieri VAN BENEDEN und die Verbreitung der planktischen Stadien in der südlichen Nordsee (Athecatae-Anthomedusae). Helgoländer wiss. Meeresunters. 5, 234-250.

KuHL, W., 1966. Eine mikrokinematographische Vielzweckeinrichtung für 16-mm-Schmalfilm. Z. wiss. Mikrosk. 67, 206-224.

Kurt, W. \& Kuнt, G., 1967. Zur Dynamik der Entwicklung yon Ectoplettra dumortieri (Athecatae-Anthomedusae) bis zur Proactinula. Laufbild- und Teilbildanalysen von Mikrozeitrafferfilmaufnahmen. Helgoländer wiss. Meeresunters. 16 (im Druck). 